\title{
Nonstretch normal moveout through iterative partial correction and deconvolution
}

\author{
Ettore Biondi ${ }^{1}$, Eusebio Stucchi ${ }^{2}$, and Alfredo Mazzotti ${ }^{3}$
}

\begin{abstract}
Source to receiver distances used in seismic data acquisition have been steadily increasing and it is now common to work with data acquired with more than $10 \mathrm{~km}$ of offset. Subbasalt exploration and seismic undershooting are just two applications in which long-offset reflections are sought. However, such reflections are often subjected to muting to suppress normal moveout (NMO) stretch artifacts, thus causing a loss of valuable information. To retrieve these portions of the recorded wavefield, we developed a nonstretch NMO correction based on wavelet estimation and on an iterative procedure of partial NMO correction and deconvolution. We evaluated this methodology using fourth-order traveltime curve approximations to
\end{abstract}

increase the offset of usable reflections, but it can be adapted to traveltime curves of any order. Time- and space-variant wavelets, estimated by means of singular value decomposition along the sought traveltimes, were used to build the desired output for the deconvolution that aims at retrieving the original shape of the partially stretched wavelets. We tested our method on a synthetic gather presenting time and offset varying wavelets, on a real-marine line simulating an undershooting pattern and on true undershooting land-marine data. These examples demonstrated that our new algorithm effectively limits the stretching associated with the NMO correction and enables the recovery of those portions of the stacked sections that are typically lost from muting in the standard NMO correction.

\section{INTRODUCTION}

Normal moveout (NMO) correction is a crucial processing phase needed to remove the offset and velocity effects present in a common midpoint (CMP) gather. The result of this operation generates a CMP gather where all the traces, recorded at variable source to receiver offset, simulate a zero-offset kinematic. The data are then used for stacking or for amplitude variations with offset (AVO) analysis. Unfortunately, the traditional sample-by-sample NMO correction introduces the stretching of the reflected wavelets (Buchholtz, 1972; Dunkin and Levin, 1973). These distortions are due to the nonparallelism of the local traveltime of each reflection event (Perroud and Tygel, 2004; Masoomzadeh et al., 2010) or in the frequency domain to the nonphysical energy changes caused by the nonstationary time shifts applied (Barnes, 1992; Burnett and Ferguson, 2011). Wavelet stretching is also a well-known problem that occurs in prestack time and depth migration and affects the result of the migrated images in a way that is quite similar to the conventional NMO stretch (Tygel et al., 1994; Perez and Marfurt, 2007; Zhu and McMechan, 2013).

The standard NMO correction suffers from other limitations that add to the wavelet bandwidth compression, such as the partial duplication of the recorded events and the time inversion of the samples of a reflection (Masoomzadeh et al., 2010; Zhang et al., 2013).

Because all these negative effects may distort the stacked section, the stretched part of the corrected CMP gather is usually discarded by applying a mute function computed on the basis of the maximum stretching threshold allowed. The NMO distortions worsen when the traditional correction is applied to data with a high offset-depth ratio because the application of the mute function limits the stacking process to the near-vertical reflections only. For instance, in undershooting surveys this can lead to the loss of the shallow events in the stacked section (Okaya et al., 2003; Clarke et al., 2007; Stucchi and Mazzotti, 2009).

Manuscript received by the Editor 23 October 2013; revised manuscript received 14 March 2014 ; published online 4 June 2014.

${ }^{1}$ Formerly University of Pisa, Department of Earth Sciences-Geophysics, Pisa, Italy; presently University of Milan, Department of Earth SciencesGeophysics, Milan, Italy. E-mail: ettore.biondi@unimi.it.

${ }^{2}$ University of Milan, Department of Earth Sciences-Geophysics, Milan, Italy. E-mail: eusebio.stucchi@unimi.it.

${ }^{3}$ University of Pisa, Department of Earth Sciences-Geophysics, Pisa, Italy. E-mail: mazzotti@dst.unipi.it.

(C) 2014 Society of Exploration Geophysicists. All rights reserved. 
In exploration seismology, it is now common to acquire and process data with more than $10 \mathrm{~km}$ of offset (see, e.g., Colombo, 2005; Maili et al., 2013). There are various reasons for long-offset acquisition. In subbasalt exploration, wide-angle data are used to provide information on the reflectors beneath the basalt layers (White et al., 2003; Maresh and White, 2005). In these geological settings, wideangle amplitudes can be significantly higher than the amplitudes of the near-vertical incidence reflections. Furthermore, long-offset reflected events are not obscured by long-period multiples generated from the seabed and from top-basalt reflectors (Masoomzadeh et al., 2010). The recovery of the long-offset wavefield is also extremely important in undershooting survey. Here, wide-angle acquisition geometry let us record raypaths that have not traversed complicated bodies such as salt domes, providing more useful seismic images for interpretation (Bading et al., 1974; Moldoveanu et al., 1993). The undershooting technique is also used when short-offset sources or receivers cannot be lain down due to the presence of drilling platforms in exploration seismology or other obstacles in shallowseismic surveys (Howdeshell, 1986; Duncan et al., 1989; Stucchi and Mazzotti, 2009). Beside these applications, wide-angle reflections are used in AVO analysis to improve the accuracy of parameters' estimation (Drufuca and Mazzotti, 1995; Roberts, 2000; Downton and Ursenbach, 2006), and for creating starting models for full-waveform inversion (Prieux et al., 2013).

The block-move-sum (BMS) method (Rupert and Chun, 1975) is one of the earliest alternative algorithms that deals with NMO stretch. In the BMS method, the samples of each trace are gathered into overlapping blocks, which are rigidly moved to the correct time. These blocks are then weighted and summed to form the NMO-corrected gather. The main problem of the BMS algorithm is the introduction of spurious wavelets that are a replica of the reflected events. A similar approach, based on the assumption that all the time samples of a reflected wavelet have the same NMO correction, is proposed by Shatilo and Aminzadeh (2000). Their method requires that the processing analyst picks all the events that are to be stacked without stretch; however, this operation can be quite demanding in a commercial exploration seismic line. Brouwer (2002) extends the BMS technique using time varying blocks and applies a coherence filter to compensate for the specific artifacts thus introduced. All these three block-based methods are affected by the possible introduction of horizontal coherent artifacts that, after stacking, can mimic true reflected events.

Lichman (1999) considers the NMO to be a process that involves only the phase spectra of the recorded wavefield and introduces the phase-based moveout algorithm. In this procedure, the phase spectrum of each finite-offset trace is replaced with the phase spectrum of the minimum-offset trace. Because the amplitude spectrum of each corrected trace is preserved, high-frequency content is maintained. However, the estimation of the phase spectrum of the minimum-offset trace is extremely sensitive to the signal-to-noise ratio $(\mathrm{S} / \mathrm{N})$ of the near-offset traces and thus the results depend on the quality and fold of the recorded data. Hicks (2001) describes a method for removing NMO during stacks that uses the parabolic radon transform properties. He also develops a technique that removes stretch from a corrected CMP gather based on a combination of radon and spatial Fourier transforms. The downside of this procedure is that the processing analyst must pick an appropriate path in the radon domain, or rely on an automatic picker whose reliability in the face of noise is questionable. Moreover, Hicks's and Lichman's techniques do not account for interfering events and may introduce horizontal-coherence artifacts.

Trickett (2003) describes a stretch-free stacking method, in which the NMO correction and the stack phase are replaced by a singlestep inversion that generates the zero-offset stacked trace. A characteristic of this procedure is that an NMO-corrected CMP gather useful for AVO analysis is never formed. Hunt et al. (2003) partly overcome this limit by calculating a series of offset-limited stretchfree stacks.

Perroud and Tygel (2004) propose a method based on a different concepts than that of the other authors. The main idea is to maintain the parallelism of the local traveltime to avoid wavelet distortions. They accomplish this by modifying the velocity profile of each CMP and thus changing the asymptote of each local traveltime. Their nonstretch NMO has the limitation of using the hyperbola approximation for the reflection kinematic, and this makes the procedure less applicable to data with high offset-depth ratio. On the basis of velocity profile modification, Masoomzadeh et al. (2010) develop a method that uses isomoveout curves (line of equal moveout) in the time-velocity panel to achieve the local traveltime parallelism for the selected reflections. The result of this operation is a zig-zag velocity function that is used for the NMO corrections. This method is affected by two main drawbacks: the processor analyst must pick the events to be corrected without distortions and the steep change in the zig-zag velocity field could create discontinuities in the corrected gathers. Recently, Zhang et al. (2013) introduce a nonstretch technique based on an iterative-matchingpursuit algorithm, which recovers wavelet distortions caused by NMO correction. Although their method can retrieve most of the recorded wavefield, it dampens the wavelet amplitudes of crossing events.

We present an extension of the algorithm for NMO through iterative partial correction and deconvolution (Mazzotti et al., 2005), which deals with data characterized by high offset-depth ratio and offset varying waveforms. We test our method on a synthetic seismic gather that presents long-offset traces, interfering events, and amplitude and phase variations of the reflected waveforms. We also use the new method on two different real seismic data sets. In the first application, we consider an offshore marine line (Rocchi et al., 2007) and we simulate an undershooting pattern by muting the short-offset traces. Then, we compare the results obtained using the full-offset data and the data obtained by the simulated undershooting. In the second real data example, we apply the iterative partial correction algorithm to an undershooting land-marine line, where the shallow part of the stacked section is completely zeroedout by the muting of the overstretched wavelets in the NMOcorrected CMPs (Stucchi and Mazzotti, 2009).

\section{NMO THROUGH PARTIAL CORRECTION}

The idea behind NMO through partial correction (NMOPC) is that each reflected event is only partially NMO corrected and then the limited distortions introduced by this operation are removed by means of Wiener-shaping deconvolution. The desired output for the filter computation is built using the waveforms estimated along the traveltime curves derived from the velocity analysis. In our implementation, we make use of the fourth-order approximation but other traveltime curves could be adopted as well. The wavelet estimation is carried out by applying singular value decomposition (SVD) on 
the data within spatial and temporal windows that slide on the original data gather.

The NMOPC algorithm is divided into three main parts: wavelet estimation, partial NMO correction, and deconvolution. We will describe each part after a short review of the traveltime approximation in a flat-layered earth.

\section{High-order traveltime approximation}

The earliest traveltime equation used for NMO correction is the well-known hyperbolic approximation (Dix, 1955) for flat-layered media:

$$
t\left(t_{0}, x\right)=\sqrt{t_{0}^{2}+\frac{x^{2}}{V_{\mathrm{rms}}^{2}\left(t_{0}\right)}}
$$

where $t_{0}$ is the two-way traveltime at zero-offset, $x$ is the source to receiver offset, and $V_{\text {rms }}\left(t_{0}\right)$ is the root-mean-square velocity.

In data characterized by a high offset-depth ratio, the hyperbolic approximation cannot reproduce long-offset traveltime with high accuracy (Castle, 1994). To deal with these data, the NMOPC uses the fourth-order equation described in Taner and Koehler (1969):

$$
\begin{aligned}
t\left(t_{0}, x\right) & =\sqrt{t_{0}^{2}+\frac{x^{2}}{V_{\mathrm{rms}}^{2}\left(t_{0}\right)}+\frac{V_{\mathrm{rms}}^{4}\left(t_{0}\right)-V_{4}^{4}\left(t_{0}\right)}{4 t_{0}^{2} V_{\mathrm{rms}}^{8}\left(t_{0}\right)} x^{4}} \\
& =\sqrt{c_{1}+c_{2} x^{2}+c_{3} x^{4}},
\end{aligned}
$$

where traveltime $t$ is a function of the two-way traveltime at zerooffset $t_{0}, x$ is the offset, $V_{\text {rms }}$ is the root-mean-square velocity, and $V_{4}$ is the quartic velocity (Castle, 1994).

To retrieve the quartic velocities, we perform a Dix conversion of the rms velocities into interval velocities and then compute the quartic velocity up to layer $N$ by means of

$$
V_{4}=\sqrt[4]{\frac{\sum_{i=1}^{N} \Delta t_{i} V_{i}^{4}}{\sum_{i=1}^{N} \Delta t_{i}}},
$$

where $V_{i}$ is the interval velocity of the $i$ th layer and $\Delta t_{i}$ is the vertical traveltime of the $i$ th layer. The only input needed for this procedure are the time-velocity peaks obtained from the analysis of conventional velocity spectra, linearly interpolated to obtain sample-by-sample values.

We test this procedure on the layered-earth model shown in Table 1 . From the $t_{0}$-rms velocity pairs of the synthetic seismogram computed using the Červený (1985) package, the coefficients of the fourth-order approximation can be retrieved by means of equation 3 . To estimate the error due to the hyperbolic traveltime and the fourth-order Taner and Koehler's series approximation, we calculated the ray-traced traveltime for each reflection (Figure 1). As is well known (Castle, 1994), the hyperbolic approximation is accurate to an offset about equal to the depth of the reflector, after which it diverges from the true traveltime (red dotted-dashed line). Conversely, the fourth-order equation accurately reproduces the ray-traced traveltime for source-to-receiver distances greater than the depth of the reflector (blue dashed line).

\section{Wavelet estimation}

Estimating wavelets from observed data is a delicate issue, which entails a degree of subjective judgment and can be carried out in different ways, such as averaging or least-squares fitting reflected waveforms of different traces or by applying eigenimage decomposition (SVD) to the selected data matrix (Lines and Treitel, 1984; Kirlin and Done, 1999). We choose the SVD method because it is easily applicable and can be adapted to different kinds of data (different number of traces, $\mathrm{S} / \mathrm{N}$, temporal window length and so on) with no additional effort.

We use temporal-offset estimation windows that slide in time and offset. The short-offset window (window 1 in Figure 2a) is moved along the fourth-order curve corresponding to its central time sample (Figure 2a). These windows enable us to select portions of the wavefield where the reflected wavelets can be considered as stationary and define a matrix in which to apply the SVD algorithm for the wavelet estimation. Usually, the first eigenimage allows a reconstruction of the main features of the wavelet within each temporal-offset gate, whereas in more complicated cases (such as when the reflections are not perfectly aligned along the sought traveltimes), higherorder eigenimages may be required. This is typically a subjective choice that requires a few trial-and-error attempts to be defined.

As an example, in Figure 2b, we show the representative wavelets for the last two overlapped reflections of Figure 2a computed as the average of the traces of the first eigenimage for each window. We used a total of 22 offset windows composed by nine traces that were successively time shifted. In our applications, the time shift ranges between $50 \%$ and $20 \%$ of the window time length. In this case, we time shifted the estimation windows by half of their temporal length and repeated the whole procedure (Figure $2 \mathrm{a}$ ). The estimation process starts from the first $t_{0}$ sample and covers the whole time axis. Thus, we end up with a representative wavelet $w\left(t_{i}, x_{j}\right)$ for each time-offset window, which follows the fourth-order traveltime curves.

To avoid edge effects on the representative waveforms $w\left(t_{i}, x_{j}\right)$ the estimation windows are time and offset partially overlapped. The time overlap depends on the time shift, as described above, whereas the offset superposition ranges between $20 \%$ and $50 \%$ of the offset width of the window.

From our tests, we find that the best result for the estimation process and the successive unstretched CMP creation is achieved using a

Table 1. Flat-layered earth model used to compute the traveltime residuals shown in Figure 1 and the synthetic gather of Figures 2a and 5a.

\begin{tabular}{llll} 
Layer thickness & P-wave velocity & \multicolumn{1}{c}{ S-wave velocity } & Density \\
\hline$h_{1}=400 \mathrm{~m}$ & $V_{\mathrm{P} 1}=1500 \mathrm{~m} / \mathrm{s}$ & $V_{\mathrm{S} 1}=0 \mathrm{~m} / \mathrm{s}$ & $\rho_{1}=1000 \mathrm{~kg} / \mathrm{m}^{3}$ \\
$h_{2}=200 \mathrm{~m}$ & $V_{\mathrm{P} 2}=1700 \mathrm{~m} / \mathrm{s}$ & $V_{\mathrm{S} 2}=303.57 \mathrm{~m} / \mathrm{s}$ & $\rho_{2}=1200 \mathrm{~kg} / \mathrm{m}^{3}$ \\
$h_{3}=700 \mathrm{~m}$ & $V_{\mathrm{P} 3}=1600 \mathrm{~m} / \mathrm{s}$ & $V_{\mathrm{S} 3}=761.90 \mathrm{~m} / \mathrm{s}$ & $\rho_{3}=1900 \mathrm{~kg} / \mathrm{m}^{3}$ \\
$h_{4}=500 \mathrm{~m}$ & $V_{\mathrm{P} 4}=2000 \mathrm{~m} / \mathrm{s}$ & $V_{\mathrm{S} 4}=1111.11 \mathrm{~m} / \mathrm{s}$ & $\rho_{4}=2000 \mathrm{~kg} / \mathrm{m}^{3}$ \\
$h_{5}=1000 \mathrm{~m}$ & $V_{\mathrm{P} 5}=2100 \mathrm{~m} / \mathrm{s}$ & $V_{\mathrm{S} 5}=1300 \mathrm{~m} / \mathrm{s}$ & $\rho_{5}=2200 \mathrm{~kg} / \mathrm{m}^{3}$ \\
$h_{6}=50 \mathrm{~m}$ & $V_{\mathrm{P} 6}=2400 \mathrm{~m} / \mathrm{s}$ & $V_{\mathrm{S} 6}=1500 \mathrm{~m} / \mathrm{s}$ & $\rho_{6}=2500 \mathrm{~kg} / \mathrm{m}^{3}$ \\
$h_{7}=\infty$ & $V_{\mathrm{P} 7}=3000 \mathrm{~m} / \mathrm{s}$ & $V_{\mathrm{S} 7}=1875 \mathrm{~m} / \mathrm{s}$ & $\rho_{7}=2800 \mathrm{~kg} / \mathrm{m}^{3}$ \\
\hline
\end{tabular}



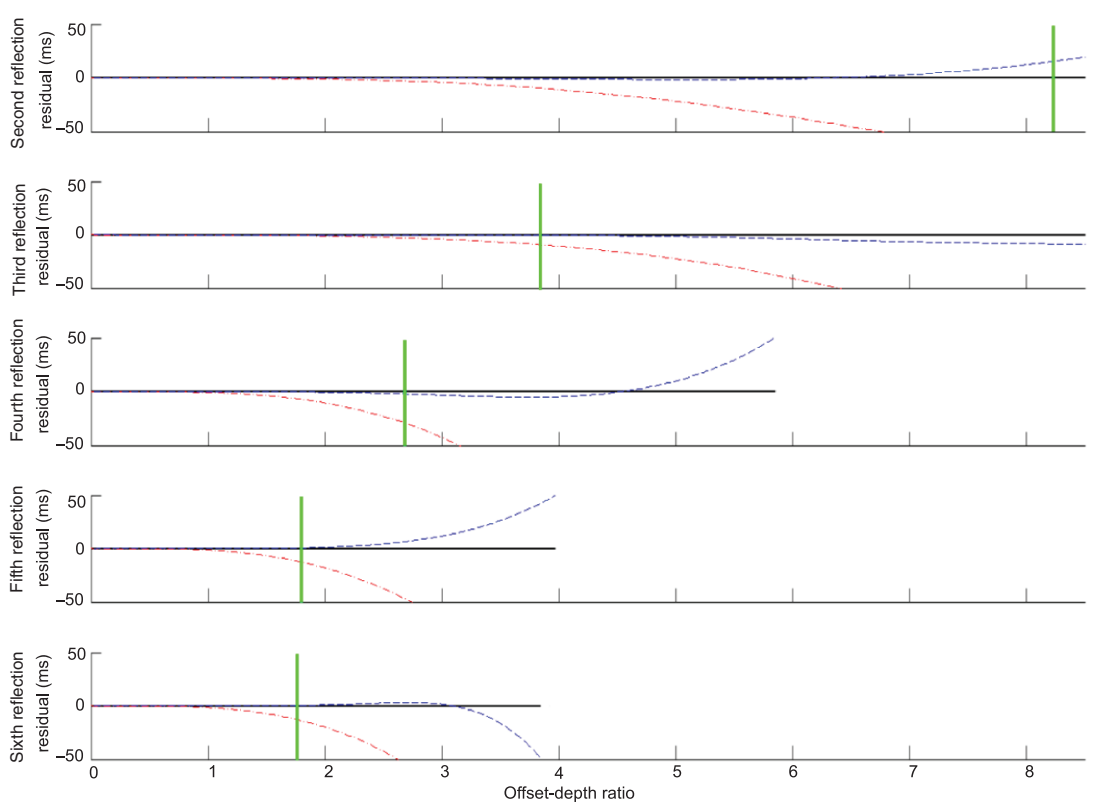

Figure 1. Traveltime residuals of the hyperbolic approximation (red dotted-dashed line, equation 1) and of the fourth-order approximation (blue dashed line, equation 2) with respect to the ray-traced traveltimes. The green bars on each residual indicate an offsetdepth ratio that corresponds to a source-receiver distance of $5000 \mathrm{~m}$ (the longest offset of the synthetic gather of Figures $2 \mathrm{a}$ and $5 \mathrm{a}$ ).

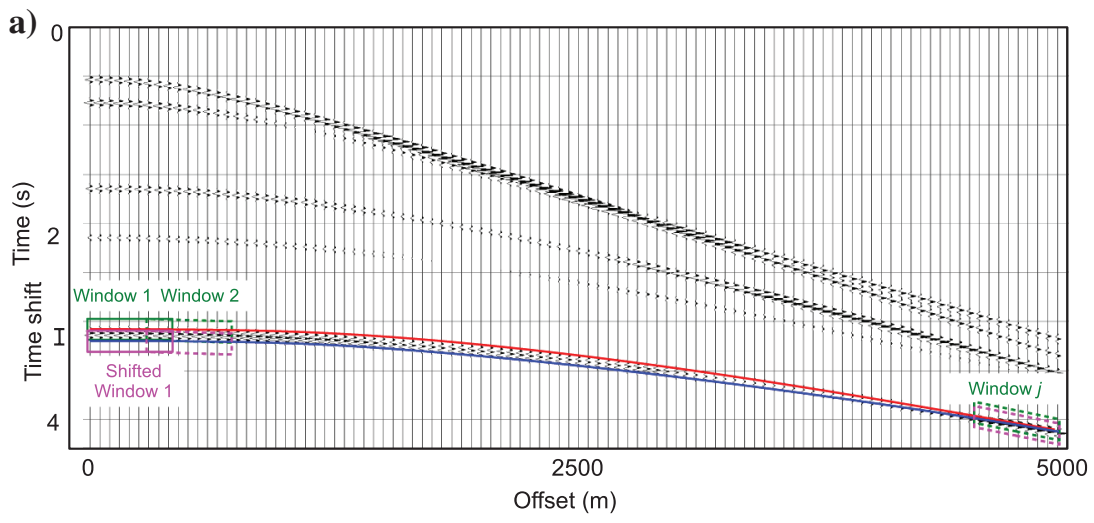

b)

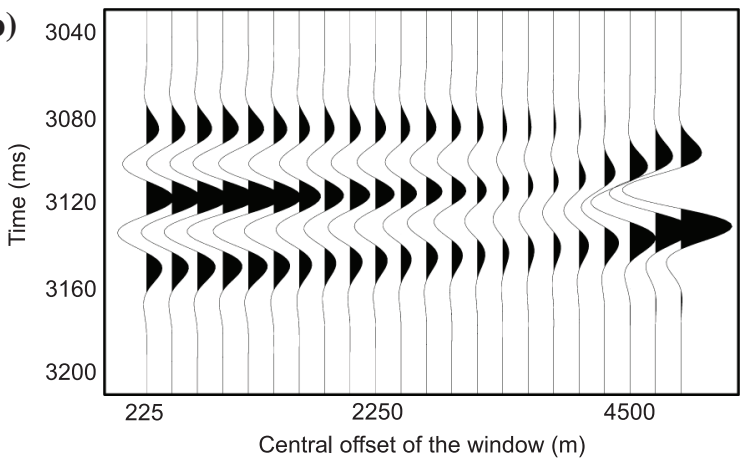

Figure 2. Wavelet estimation process with time-offset windows sliding in offset and time. (a) Estimation windows centered along the fourth-order curve with $t_{0}=3120 \mathrm{~ms}$ (red line). The short-offset window (green box) is then time shifted (magenta box) and the sliding procedure for the wavelet estimation along the new fourthorder traveltime curve (blue line) is repeated. The time shift is half the window time length and (b) estimated waveforms after the application of the SVD on 22 offset windows that have been moved along the fourth-order curve with $t_{0}=3120 \mathrm{~ms}$. window with time dimension comparable to the length of the source wavelet. The offset dimension depends on whether the waveform variations along the offset are strong or mild. In the former case, a narrow-offset window is necessary, whereas in the latter case it is possible to use a wider-offset gate.

The efficacy of the SVD estimation process strictly depends on the wavelet alignment within the estimation matrices. Thus, for data with high offset-depth ratio, it is necessary to use a high-order traveltime approximation to extract a reliable and representative wavelet from the wide-angle estimation windows.

\section{Partial correction}

The second phase of our method is a partial NMO correction. Its purpose is to limit the waveform distortions, which would be greater if the corrections were performed in a single step. We regulate the amount of NMO correction by means of the NMO percentage parameter that is defined as follows:

$$
\alpha=\frac{t_{1}-t_{1 \text { new }}}{t_{1}-t_{0}},
$$

where $t_{1}$ is the traveltime at a reference offset (such as the farthest available offset) for the uncorrected trajectory (red line in Figure $3 a), t_{\text {1new }}$ is the traveltime at the same offset for the partially corrected trajectory (cyan line in Figure $3 b$ ), and $t_{0}$ is the two-way vertical traveltime (green line in Figure 3a and 3b). The typical value of $\alpha$ ranges between $30 \%$ and $40 \%$.

Once $\alpha$ has been decided, it is possible to move the original traveltime curve $t_{\text {old }}$ to the partially NMO corrected trajectory $t_{\text {new }}$ by means of the following equations:

$$
\begin{gathered}
\beta=\frac{t_{\text {1new }}^{2}-t_{0}^{2}}{c_{\text {2old }} \bar{x}^{2}+c_{\text {3old }} \bar{x}^{4}}, \\
t_{\text {new }}(x)=\sqrt{t_{0}^{2}+\beta c_{\text {2old }} x^{2}+\beta c_{3 \text { old }} x^{4}},
\end{gathered}
$$

where $c_{2 \text { old }}$ and $c_{3 \text { old }}$ are the coefficients of the fourth-order equation $2, \bar{x}$ is a reference offset, and $x$ is the offset. The result of the partial correction is a CMP, in which the reflections have been only partially moved to their final horizontal alignment so that they have been subjected to minimal stretch. For example, Figure $3 b$ shows the partial correction of the reflection in Figure 3a, with a NMO percentage of $35 \%$ referred to the maximum offset of $4950 \mathrm{~m}$. The number of iterations needed to achieve a complete NMO correction is determined by 
the NMO percentage. On the one hand, a value that is too high introduces unrecoverable wavelet distortions. On the other hand, a value that is too low makes our procedure computationally expensive. Thus, the number of iterations is a trade-off between the introduced distortions and the computational cost. For instance, in the reported applications, we use a NMO percentage that varies between $30 \%$ and $40 \%$ corresponding to several iterations from four to three.

In the examples we discuss, we make use of the fourth-order Taner and Koehler (1969) approximation. To extend the kinematic description to higher-order traveltime curves, we have to include in the $\beta$ coefficient the offset terms up to the desired order using the following relation:

$$
\beta=\frac{t_{1 \text { new }}^{2}-t_{0}^{2}}{c_{\text {2old }} \bar{x}^{2}+c_{3 \mathrm{old}} \bar{x}^{4}+c_{4 \mathrm{old}} \bar{x}^{6}+\cdots},
$$

and multiply $\beta$ with all the $c_{i}$ terms in the Taner and Koehler series. This procedure of partial correction can be applied to any $t\left(t_{0}, x\right)$ moveout function, therefore extending the kinematic approximation to the desired order.

\section{Shaping deconvolution}

Distortion caused by the partial NMO correction can be removed by applying Wiener filters shaping the stretched wavelets to their original (unstretched) status. By knowing the relation between the old and the new traveltime curves (equation 6), it is possible to build the partially corrected unstretched CMP gather, which constitutes the desired output. To this goal, the estimated wavelets $w\left(t_{i}, x_{j}\right)$ are placed along the corresponding fourth-order traveltime curves starting from offset zero and time zero. This procedure is carried out using the same window lengths and overlaps as those used in the estimation phase. To dampen spurious oscillations in the unstretched CMP caused by the window overlap, we apply a time and offset Hamming window.

Once the desired output has been created, we deconvolve the partially corrected and stretched CMP with Wiener filters of about the same length as the source wavelet. Because the wavelet stretching depends on offset and time, we design various time windows and we apply different deconvolution operators on a trace-by-trace and window-by-window basis. The time length of these windows is selected depending on the record length and on the amount of stretching due to the partial correction. In general, these deconvolution gates are much longer than the windows used for estimating the wavelets.

The procedure of partial NMO correction and shaping deconvolution is then reiterated until a complete NMO correction is achieved. Figure 4 shows the flowchart of the NMOPC algorithm.

\section{SYNTHETIC DATA APPLICATION}

Using a ray-tracing algorithm (Červený, 1985) on the earth model of Table 1, we computed the synthetic CMP gather shown in Figure $2 \mathrm{a}$ and replotted in Figure 5a for the NMOPC test. This synthetic example is similar to the one shown in Zhang et al. (2013). Six reflections with amplitude and phase variations with offset are present; of these events, the latter two almost overlap due to the small thickness of the sixth layer (50 m only). In this example, the trajectory for the second reflection deviates strongly from its hyperbolic approximation (note the size of the residuals at the green bar in Figure 1).

Figure $5 \mathrm{~b}$ shows the NMO-corrected synthetic data with no mute applied. The conventional NMO correction, on a sample-by-sample basis, was performed considering the exact fourth-order traveltime curves. Several well-known artifacts can be observed in this image. First, the two shallower reflections are affected by significant overstretching and distortions in the interfering part, making them unusable for offsets greater than approximately $800 \mathrm{~m}$. Second, the traveltime curves which do not correspond to a reflected event, but are nevertheless used by the NMO procedure, cause wavelet

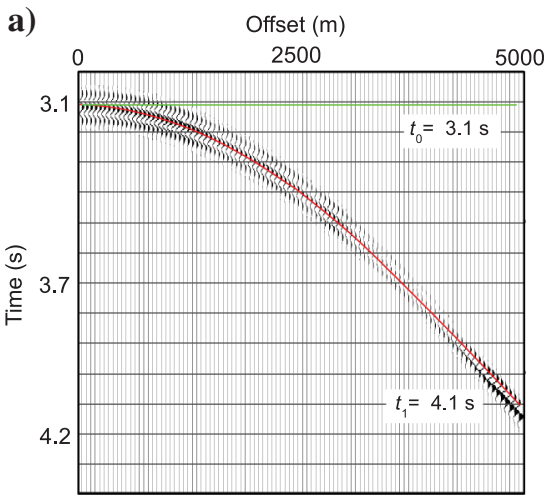

b)

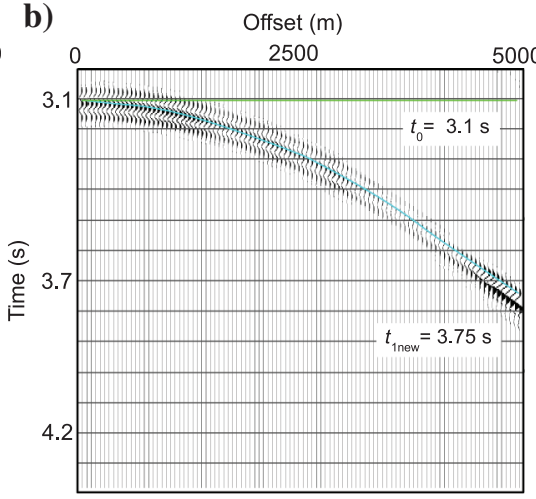
5000

Figure 3. Partial correction applied to the synthetic data. The uncorrected event lies on the red line (a) then, after partial correction with $\alpha=35 \%$, it is moved to the blue line (b). The waveforms at long-offset are only partially distorted.

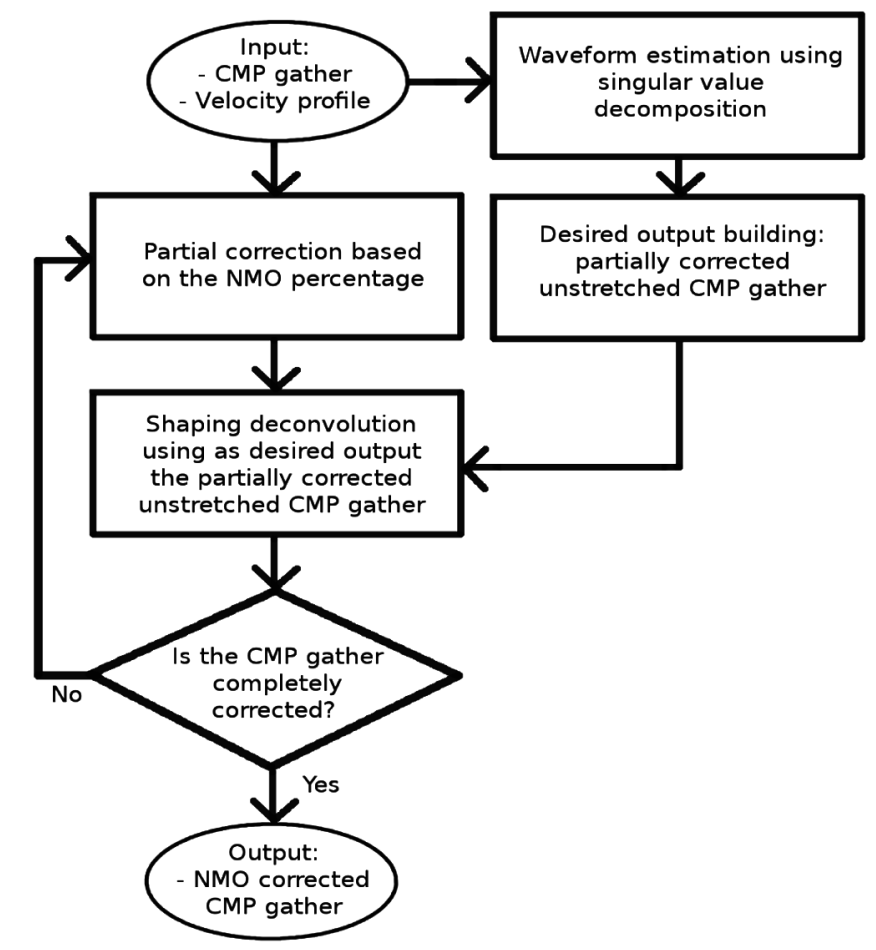

Figure 4. Flowchart of the NMOPC algorithm. 
a) 0

Offset (m)

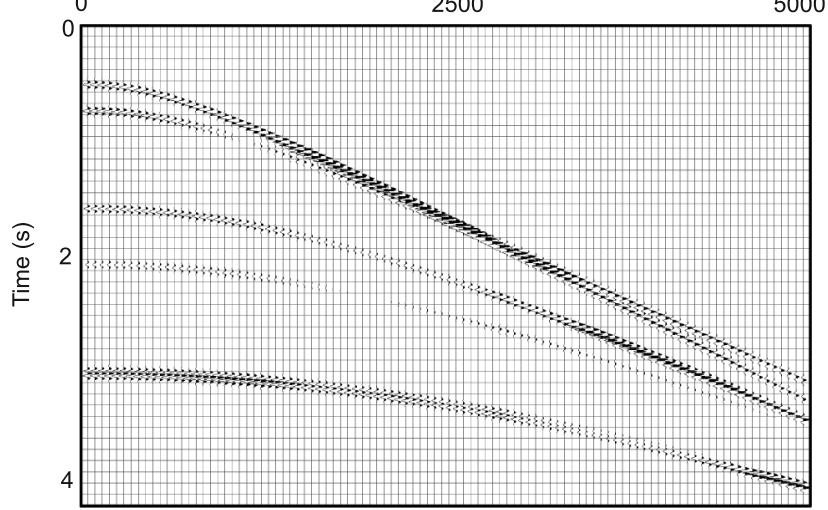

b)

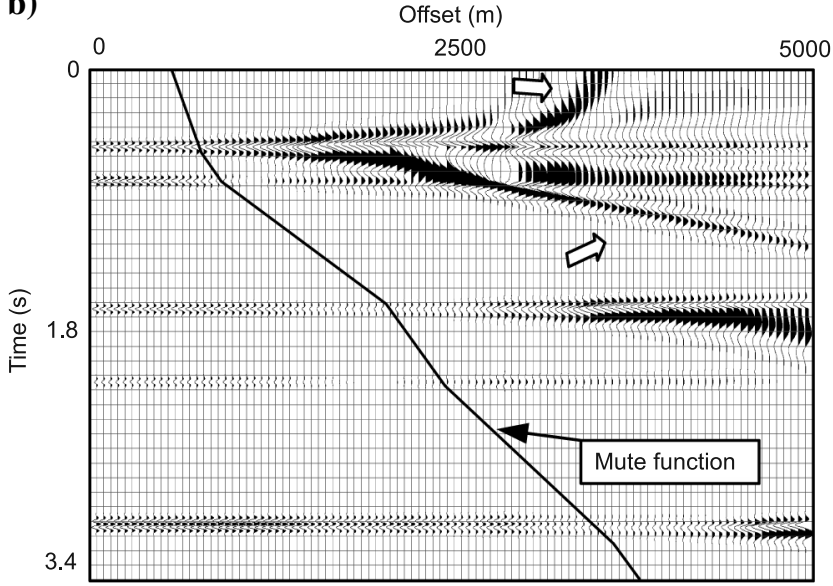

c)

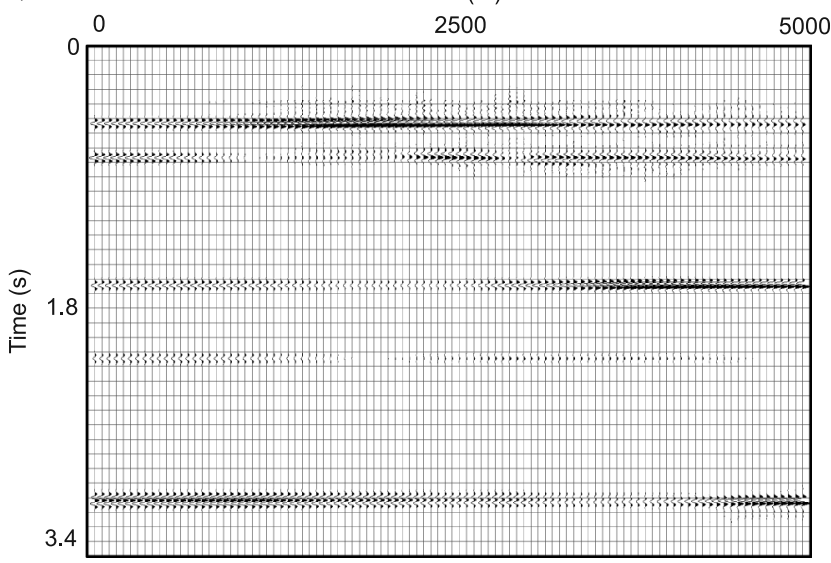

Figure 5. (a) Synthetic gather obtained using a ray-tracing routine that considers waveform variations with offset and (b) the same gather after the application of conventional NMO correction but following the exact fourth-order traveltimes. A great portion of the recorded wavefield is distorted. Moreover, duplicated events appear, as indicated by the arrows. The application of a mute function with stretching factor of 1.3 (black line) would cause the loss of many waveforms especially for the shallow events. (c) The same gather after the NMOPC correction. Interfering events are now separated, and the stretching is removed. Moreover, the shape and amplitude of the waveforms seem to be preserved. repetitions to appear, as indicated by the arrows in the corrected panel. Finally, the other reflections are affected by waveform stretching particularly at the far offsets.

The application of the stretching mute would cause the loss of many parts of the corrected gather, especially for the first two events (see the mute function indicated by the black line in Figure 5b). In contrast, in the CMP corrected with the NMOPC shown in Figure $5 \mathrm{c}$, the stretching and distortions of the corrected events are limited. This allows us to keep the most of the data (in this case up to the farthest offset) while preserving the original amplitude and phase characteristics for AVO analysis and stack. The minor artifacts that do appear are caused by the filter response and are effectively attenuated by the stacking. The NMOPC parameters we selected for this example are listed in Table 2.

To show how the NMOPC works, Figure 6 illustrates the evolution of the results at each successive iteration of the algorithm. Setting the NMO percentage to 35\%, the CMP in Figure 5a is corrected in three steps. The partially corrected CMPs and the corresponding desired unstretched outputs are shown in the left and in the center columns of Figure 6, respectively. Notwithstanding the limited moveout corrections we applied, some wavelet stretching and repetitions do occur (see the small ellipses in the CMPs on the left column). However, these minor distortions are corrected by the application of proper Wiener-shaping filters as shown by the results in the right column of Figure 6. A question may arise on why we cannot directly use the desired output panels for producing the stack. Indeed, in case of simple synthetic data with few reflections as in the example shown, stacking the desired outputs would yield rather the same results as the whole proposed procedure. However, things change working with real-field data (or more complex synthetics). In fact the desired outputs are built on wavelets estimated on short temporal-offset windows making use of the first eigenimage (or of the first few eigenimages) and this produces a loss of the details at high temporal and spatial frequencies. It is the shaping deconvolution on time gates that are much larger than the wavelet estimation windows (see Table 2) that, on real data, ensures an effective removal of the stretching while preserving the fine details.

The number of iterations used to achieve the total NMO correction is a key parameter. Figure 7a shows the results for the far-offset trace $(4950 \mathrm{~m})$ of the NMOPC application using one, two, or three partial corrections. As the number of iterations increases the NMOPC output converges to the desired ray-traced synthetic reflected waveforms. This also happens for the short- and middle-offset traces displayed with the far-offset trace in Figure 7b. For comparison, we also plot the traces corrected with the conventional NMO (following fourth-order traveltime curves) extracted from Figure 5b. This synthetic example demonstrates that the NMOPC can deal with interfering events and amplitude and phase variations with offset.

\section{REAL DATA APPLICATIONS}

We test the NMOPC algorithm on two real seismic data sets. Table 2 lists all the parameters used in these real data examples. The first application is carried out on a marine-seismic line representing a subset of the 3D data set described in Rocchi et al. (2007). The parameters for our available data set are source interval, $50 \mathrm{~m}$; receivers interval, $12.5 \mathrm{~m}$; minimum offset, $380 \mathrm{~m}$; maximum offset, $6350 \mathrm{~m}$; and average fold coverage, 120. To test the NMOPC, we simulated an undershooting configuration, dropping the data 
acquired with offset shorter than $2300 \mathrm{~m}$ (see red line in Figure 8a). We performed standard velocity analyses before and after dropping the short-offset traces and we found that very similar velocity trends could be picked on the semblance panels.
Figure 8a shows the uncorrected CMP gather number 289. Traditional second-order NMO correction followed by a manual mute approximately corresponding to 1.3 stretching factor (the green curve in Figure 8b) results in the loss of most of the shallow-event

Table 2. NMOPC parameters used in the synthetic and real data examples.

\begin{tabular}{|c|c|c|c|c|c|c|c|c|}
\hline \multirow[b]{3}{*}{ Example } & \multirow[b]{3}{*}{ NMO $\%(\alpha)$} & \multirow{3}{*}{$\begin{array}{c}\text { Eigenimages } \\
\text { for wavelet } \\
\text { estimation }\end{array}$} & \multicolumn{4}{|c|}{ Sliding window parameters } & \multicolumn{2}{|c|}{ Deconvolution parameters } \\
\hline & & & \multicolumn{2}{|c|}{ Time } & \multicolumn{2}{|c|}{ Offset } & \multirow{2}{*}{$\begin{array}{c}\text { Filter } \\
\text { length }(\mathrm{ms})\end{array}$} & \multirow{2}{*}{$\begin{array}{l}\text { Window } \\
\text { length (ms }\end{array}$} \\
\hline & & & Length (ms) & Shift $\%$ & Width (m) & Overlap \% & & \\
\hline Synthetic & 35 & 1 & 160 & 50 & 450 & 50 & 130 & 700 \\
\hline Simulated undershooting & 40 & 2 & 40 & 35 & 400 & 40 & 35 & 2000 \\
\hline Ancona & 30 & 3 & 35 & 20 & 100 & 20 & 25 & 1000 \\
\hline
\end{tabular}
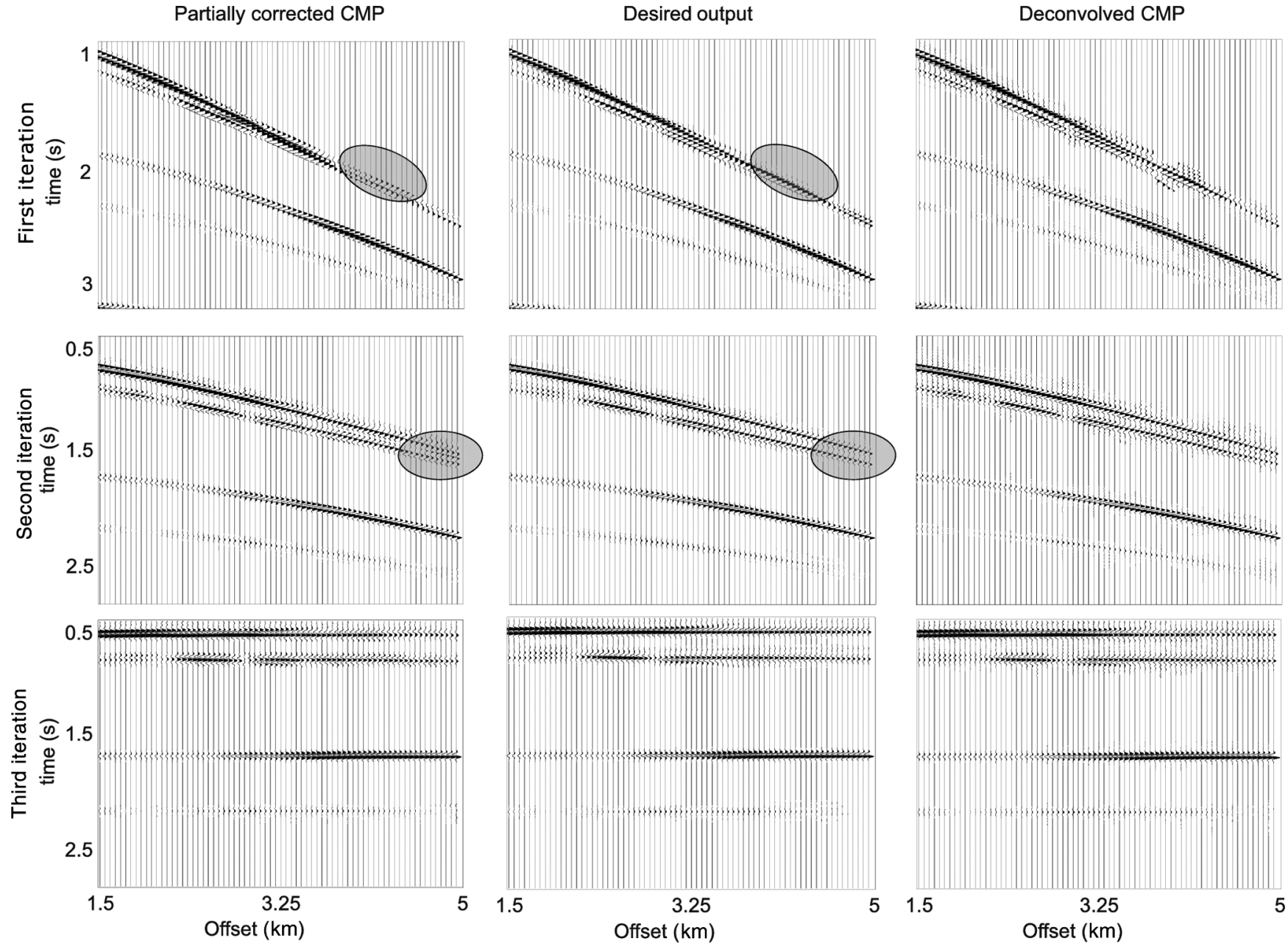

Figure 6. Results of each iteration of the NMOPC applied to the synthetic gather of Figure 5a. To facilitate the visual inspection, only the close-up of the data at shallow times and far offsets is shown. The NMO percentage $\alpha$ is $35 \%$. The panels in the left column are the partially NMO corrected CMP. Duplications and distortions, such as those within the little ellipses, appear at far offset. The panels in the center column are the unstretched and partially NMO corrected desired output used in the shaping deconvolution and built on the basis of the estimated wavelets. No duplication or significant distortions appear within the same ellipses. The right column panels are the deconvolved gathers. The deconvolved CMP of the third iteration is a portion of the entire CMP shown in Figure 5c. 
data. NMOPC, on the other hand, preserves shallow reflections and corrects long-offset waveforms (Figure 8c).

To evaluate the efficacy of the NMOPC algorithm, we built the stacked section obtained with the conventional (second-order) NMO correction using the full-offset range as a reference (Figure 9a), and the stacked sections of the undershooting test for both the traditional NMO correction (Figure 9b) and the proposed procedure (Figure 9c). In the simulated undershooting stack obtained with the conventional (second-order) NMO correction (Figure 9b) the shallowest reflections at the two sides of the section are partially deleted. The actual amount of the applied mute is strongly sensitive to time and space velocity interpolation. Furthermore, the events at approximately 2.3-2.4 s present a lower $\mathrm{S} / \mathrm{N}$ and continuity compared with the corresponding events in the full-offset stack of Figure 9a.

Conversely, in the stacked section of Figure 9c obtained by applying the NMOPC, the shallower events are preserved and there is a generally better continuity and resolution of the reflections than in Figure 9b. The improvement in resolution can be seen where the water bottom is visible in both stacks but also for the deeper reflections. Moreover, the $\mathrm{S} / \mathrm{N}$ is comparable to the full offset range section of Figure 9a, although there is a little loss of resolution.

In the second real data example, we apply the NMOPC to the land-marine records of the Ancona landslide seismic survey (Stucchi and Mazzotti, 2009). See Table 2 for the relevant NMOPC parameters. This data was acquired by using a bay-cable streamer of 48 hydrophones, anchored to the seafloor, and connected to a land acquisition system composed of 152 active channels. Shots were on land. This combined land-marine acquisition resulted in an undershooting spread, and was adopted to provide information below the coastal road and railway where recording devices could not be placed. Minimum offsets of the CMPs range between 107 and $312 \mathrm{~m}$, whereas maximum offsets vary between 323 and $444 \mathrm{~m}$.

Figure 10 illustrates the stacked sections for the three cases of conventional (second-order) NMO without mute (Figure 10a), with
Figure 7. (a) Left frame: the ray-traced synthetic reflected waveforms for the far-offset trace $(4950 \mathrm{~m})$ shifted at $t_{0}$. Right frame: output of the NMOPC as function of the number of iterations used to achieve the total NMO correction. (b) Comparison of the ray-traced waveforms (shifted at $t_{0}$ ) at 450,2450 , and $4950 \mathrm{~m}$ offset with the results obtained with the conventional NMO and with the NMOPC. The same fourth-order kinematic is used for both NMO corrections. Amplitudes and phases at the different offsets are preserved by the NMOPC algorithm.

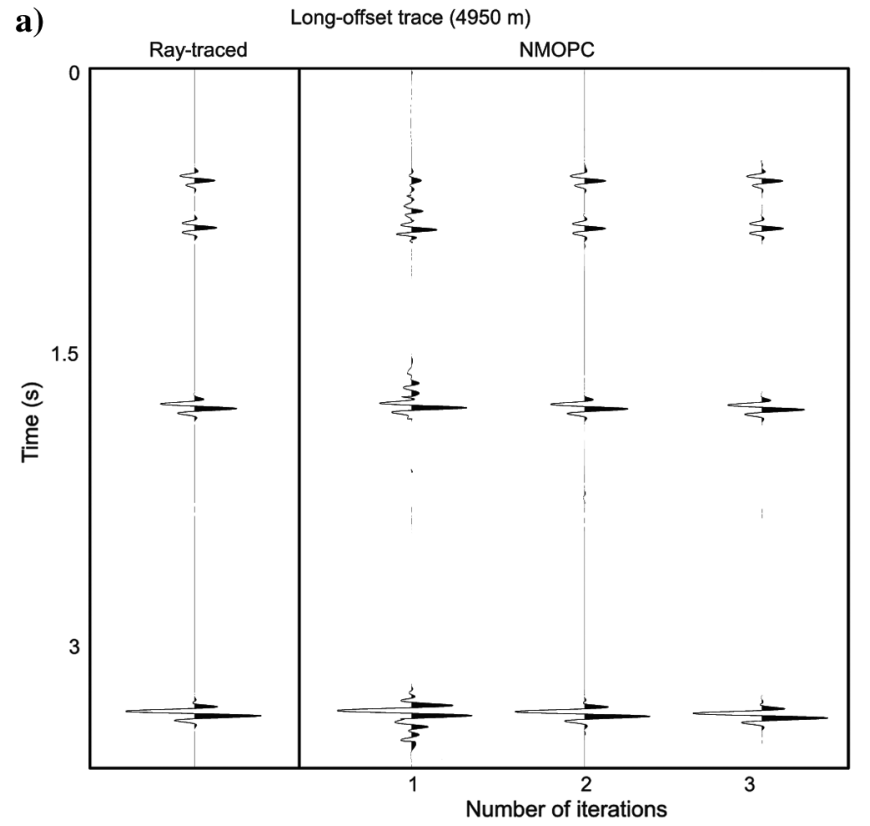

b)
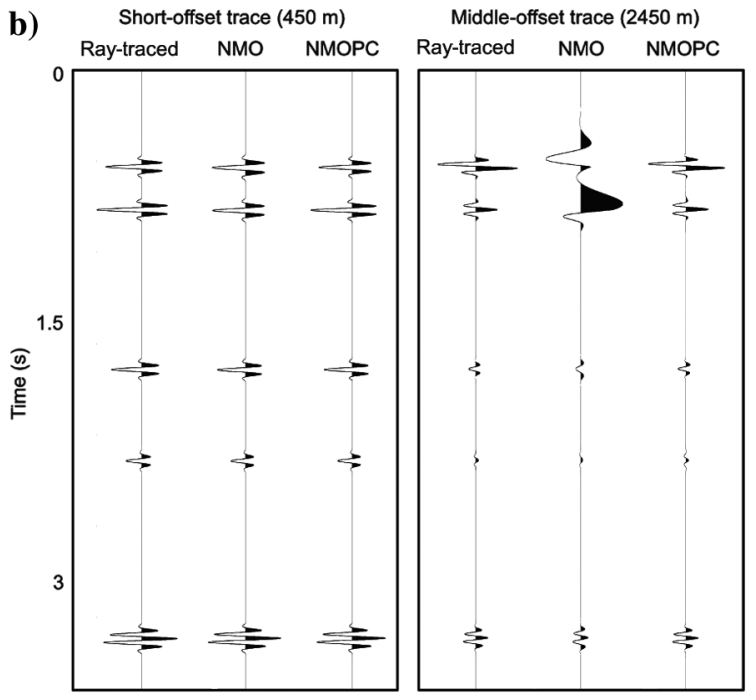

Long-offset trace $(4950 \mathrm{~m})$ Ray-traced NMO NMOPC

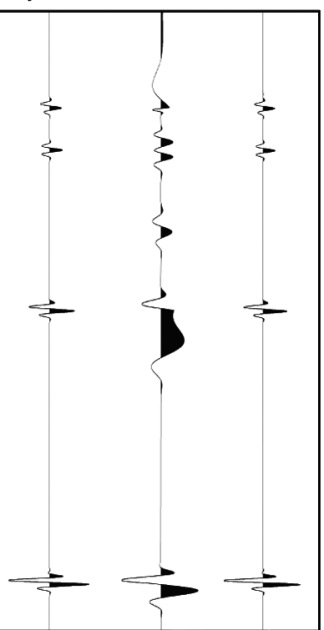


a)

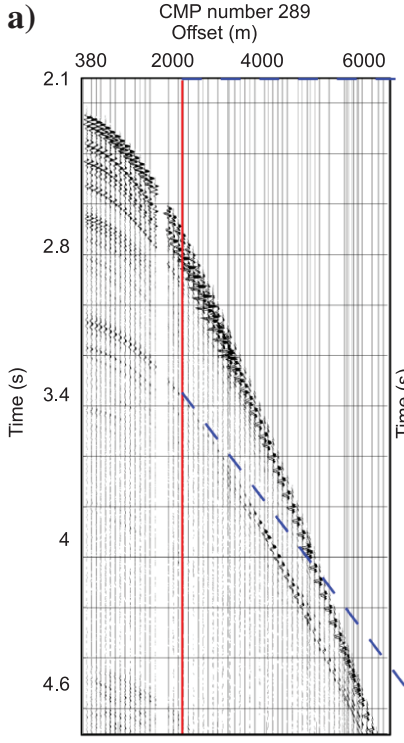

b) CMP number 289 Offset ( $\mathrm{m}$ )

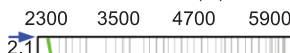

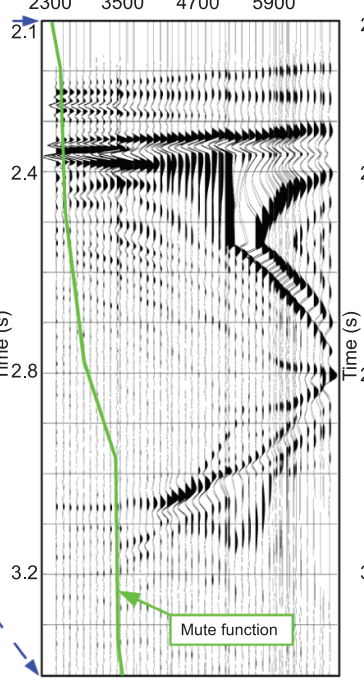

c) $\quad$ CMP number 289 Offset $(\mathrm{m})$ $2.1300 \quad 3500 \quad 4700 \quad 5900$

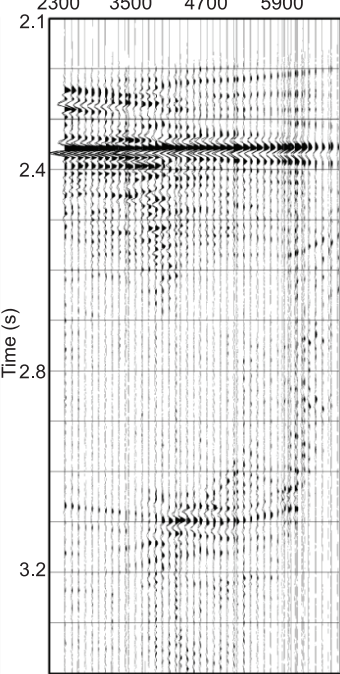

Figure 8. (a) Representative uncorrected CMP (number 289) from the data set described in Rocchi et al. (2007). The traces with offset less than $2300 \mathrm{~m}$ (red line) are dropped for simulating an undershooting acquisition. (b) Simulated undershooting CMP after the application of the conventional (second-order) NMO correction. The green line indicates the manual mute (approximately corresponding to 1.3 stretching factor) that if applied would erase all the data above the line. (c) The same CMP corrected with the NMOPC algorithm. The stretching artifacts are effectively removed at all offsets. Panels (b and c) show only the data in the offset range of the (simulated) undershooting and in a time window from 2.1 to $3.4 \mathrm{~s}$.

a)

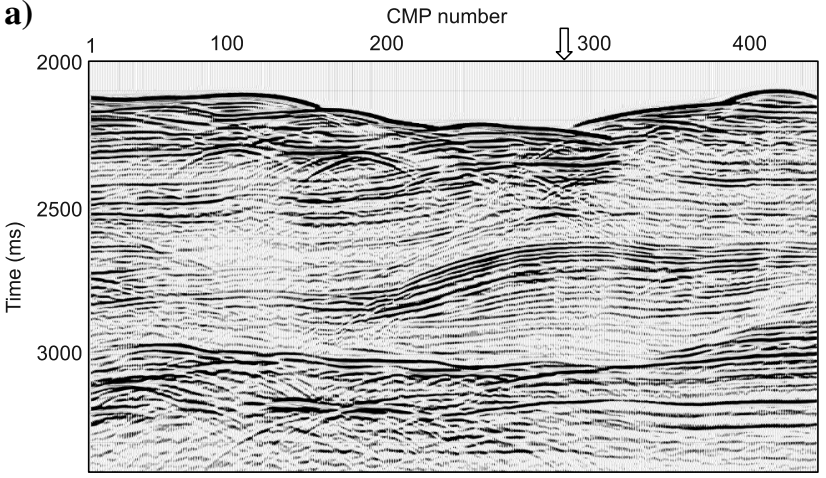

b)

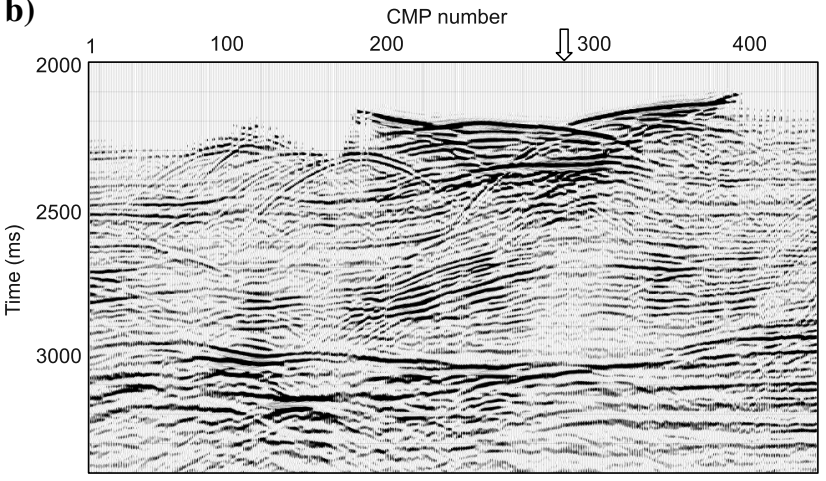

c)

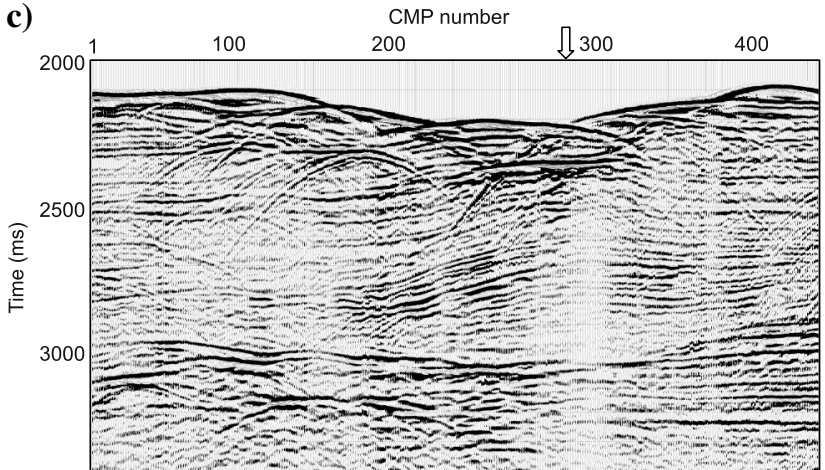

Figure 9. (a) Stacked section of a subset of the data described in Rocchi et al. (2007) using all the recorded traces and conventional (second order) NMO, (b) stacked section obtained using the simulated undershooting data after correction with the conventional NMO algorithm and manual muting such as that of Figure 8b, and (c) stacked section obtained using the simulated undershooting data after correction with the NMOPC algorithm. The NMOPC can retrieve the shallow parts of the section that are lost by applying the traditional NMO correction and muting. The arrows indicate the position of the CMP gather no. 289 of Figure 8. 
a)

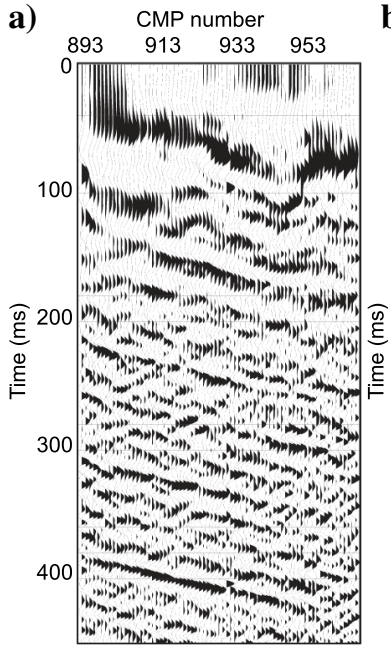

b)

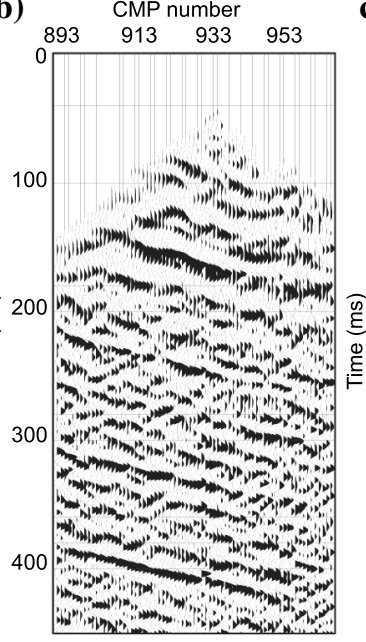

c)

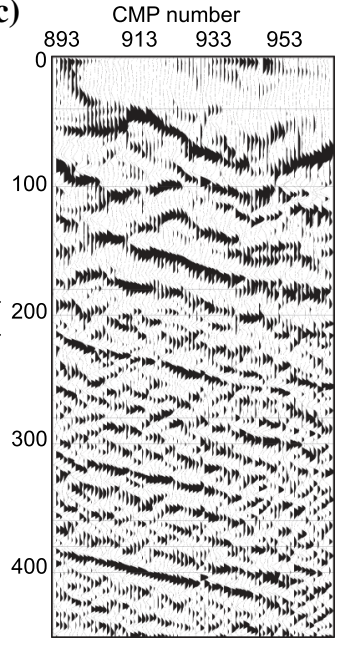

Figure 10. (a) Stacking the CMPs of the Ancona landslide data after the application of a conventional (second-order) NMO correction without any muting provides a low-resolution section in the shallow part, (b) the application of the stretching mute, although conservative, leads to partial deletion of the shallow-reflected events, and (c) correcting the CMPs using the NMOPC and then stacking the data provides a section where the foot of the landslide can be better analyzed. The reference datum for all the sections is sea level.

applied an optimized mute (Figure 10b), and after the application of NMOPC (Figure 10c)

The conventional stacked section of Figure 10a shows clear and interpretable events in the deeper part (less than $200 \mathrm{~ms}$ ), but not at shallow times (in the interval 0-140 ms), in which the stretch of the moveout correction affects the wavelets. The application of the mute function leads to the partial loss of the shallow reflections with only a minor improvement of the visibility of the events (Figure 10b). NMOPC, in contrast, provides a section (Figure 10c) with preserved shallow events that can be more easily interpreted than those of the other sections and with a resolution comparable to the conventional stack of Figure 10b. The section shown in Figure 10c might provide information on the deformation and accumulation processes occurring at the foot of the landslide that cannot be resolved with the conventional NMO correction and stacking procedure.

\section{CONCLUSIONS}

Conventional NMO correction cannot deal with recordings characterized by high offset-depth ratio and may cause overstretched signals and serious artifacts when applied to interfering events. The consequent muting of the wide-angle overstretched reflections decreases the effective coverage and limits the ability to obtain reliable stacked images, especially at shallow times.

These problems are especially serious when the useful reflections occur at large source-receiver offsets only, such as in undershooting surveys. To solve these problems, we developed the no-stretch NMO algorithm that we call NMOPC, meaning that it is based on a series of partial corrections and deconvolutions that effectively limits wide-angle wavelet stretching. The procedure is flexible and can be adapted to different data. For example, the number of estimated wavelets in time and offset can be varied according to the specific needs and targets. The same applies to the number of iterations controlled by the NMO percentage. Furthermore, the inclusion of higher-order terms in the considered traveltime curves extends the offset range of retrievable reflections. It is also feasible to apply NMOPC to prestack time-migrated gathers that have been resqueezed by a reverse NMO correction.

Results on synthetic and real data demonstrate that the NMOPC algorithm is effective in avoiding stretching effects due to NMO corrections. The experiments on synthetic data also show that amplitude and phase variations with offset of the reflected wavelets tend to be preserved by NMOPC. The simulated undershooting real data case demonstrates that the final stacked section after the NMOPC application is similar to the one obtained using the full-offset data and a standard NMO correction. In contrast, the standard NMO correction applied to the simulated undershooting data gives rise to a final stacked image with missing information in the shallow part of the section. Analogous conclusions can be drawn from the second real data experiment that consists of true undershooting acquired in a landmarine transition zone. The final stacked image after the NMOPC application is richer in fine and significant details than the conventional NMO stacked image.

As a final note, we have shown results using a particular implementation of the NMOPC but alternative schemes exist. For instance, we can set the number of iterations and leave free the NMO percentage parameter or we can limit the application of NMOPC, which is about 10 times more computationally intensive than conventional NMO, to only those shallow times where standard NMO correction is inadequate.

\section{ACKNOWLEDGMENTS}

We gratefully acknowledge the support of Landmark/Halliburton for the use of their Promax/Landmark seismic software. We also thank associate editor I. Moore for his fruitful remarks and A. E. Barnes and the other three unknown reviewers for their suggestions and constructive comments.

\section{REFERENCES}

Bading, R., J. Echterhoff, T. H. Krey, and R. Marschall, 1974, New possibilities for reflection seismics by undershooting bodies with complicated tectonics: Geophysical Prospecting, 22, 1-21, doi: 10.1111/j.1365-2478 .1974.tb00062.x.

Barnes, A. E., 1992, Another look at NMO stretch: Geophysics, 57, 749751, doi: $10.1190 / 1.1443289$.

Brouwer, J. H., 2002, Improved NMO correction with a specific application to shallow seismic data: Geophysical Prospecting, 50, 225-237, doi: 10 $.1046 / \mathrm{j} .1365-2478.2002 .00310 . x$.

Buchholtz, H., 1972, A note on signal distortion due to dynamic (NMO) correction: Geophysical Prospecting, 20, 395-402, doi: 10.1111/j $.1365-2478.1972 . t b 00642 . x$.

Burnett, W. A., and R. J. Ferguson, 2011, A reversible transform for seismic data processing: Journal of Geophysics and Engineering, 8, 477-486, doi: $10.1088 / 1742-2132 / 8 / 3 / 008$

Castle, R. J., 1994, A theory of normal moveout: Geophysics, 59, 983-999, doi: $10.1190 / 1.1443658$

Červený, V., 1985, Ray synthetic seismograms for complex two-dimensional and three-dimensional structures: Journal of Geophysics, 58, 2-26.

Clarke, R., G. Xia, N. Kabir, L. Sirgue, and S. Michell, 2007, Processing of a novel deepwater, wide-azimuth node seismic survey: The Leading Edge, 26, 504-509, doi: 10.1190/1.2723214. 
Colombo, D., 2005, Benefits of wide-offset seismic for commercial exploration targets and implications for data analysis: The Leading Edge, 24, 352-363, doi: 10.1190/1.1901383.

Dix, C. H., 1955, Seismic velocities from surface measurements: Geophysics, 20, 68-86, doi: 10.1190/1.1438126.

Downton, J., and C. Ursenbach, 2006, Linearized amplitude variation with offset (AVO) inversion with supercritical angles: Geophysics, 71, no. 5, E49-E55, doi: 10.1190/1.2227617.

Drufuca, G., and A. Mazzotti, 1995, Ambiguities in AVO inversion of reflections from a gas-sand: Geophysics, 60, 134-141, doi: 10.1190/1 .1443740 .

Duncan, P., B. Nelson, and R. Wright, 1989, Acquisition of high quality 3-D data beneath production platforms: 59th Annual International Meeting, SEG, Expanded Abstracts, 621-623.

Dunkin, J. W., and F. K. Levin, 1973, Effect of normal moveout on a seismic pulse: Geophysics, 38, 635-642, doi: 10.1190/1.1440363.

Hicks, G. J., 2001, Removing NMO stretch using the Radon and FourierRadon transforms: 63rd Annual International Conference and Exhibition, EAGE, Extended Abstracts, A-18.

Howdeshell, J., 1986, Processing of marine seismic data by two-boat undershooting: 56th Annual International Meeting, SEG, Expanded Abstracts, 360-363.

Hunt, L., S. Trickett, D. Levesque, P. McKenny, B. Link, and S. Jamieson, 2003, The effect of stretch free stacking on a clastic exploration play in Alberta, Canada: 73rd Annual International Meeting, SEG, Expanded Abstracts, 321-324.

Kirlin, R. L., and W. J. Done, 1999, Covariance analysis for seismic signal processing: SEG.

Lichman, E., 1999, Automated phase-based moveout correction: 69th Annual International Meeting, SEG, Expanded Abstracts, 1150-1153.

Lines, L. R., and S. Treitel, 1984, A review of least-square inversion and its application to geophysical problems: Geophysical Prospecting, 32, 159-186, doi: 10.1111/j.1365-2478.1984.tb00726.x.

Maili, E. A., S. Burns, N. Jones, F. Pradalie, S. Baillon, and D. Verneau, 2013, Maximizing the value of existing seismic data in the Bahrain Field with wide-azimuth processing technology: First Break, 31, 51-58.

Maresh, J., and R. S. White, 2005, Seeing through a glass, darkly: Strategies for imaging through basalt: First Break, 23, 27-33.

Masoomzadeh, H., P. J. Barton, and S. C. Singh, 2010, Nonstretch moveout correction of long-offset multichannel seismic data for subbasalt imaging: Example from the North Atlantic: Geophysics, 75, no. 4, R83-R91, doi: 10.1190/1.3443579.

Mazzotti, A., E. Stucchi, and M. Clementi, 2005, Normal moveout through partial corrections: 67th Annual International Conference and Exhibition, EAGE, Extended Abstracts, G023 Z-99.

Moldoveanu, N., R. Herman, J. Lang, and M. Chang, 1993, Undershooting using the vertical hydrophone array: The South Marsh Island experiment: 63rd Annual International Meeting, SEG, Expanded Abstracts, 536-539.

Okaya, D., T. Stern, S. Holbrook, H. van Avendonk, F. Davey, and S. Henrys, 2003, Imaging a plate boundary using double-sided onshoreoffshore seismic profiling: The Leading Edge, 22, 256-260, doi: 10 $.1190 / 1.1564531$.

Perez, G., and K. J. Marfurt, 2007, Improving lateral and vertical resolution of seismic images by correcting for wavelet stretch in common-angle migration: Geophysics, 72, no. 6, C95-C104, doi: 10.1190/1.2781619.

Perroud, H., and M. Tygel, 2004, Nonstretch NMO: Geophysics, 69, 599_ 607, doi: 10.1190/1.1707080.

Prieux, V., G. Lambaré, S. Operto, and J. Virieux, 2013, Building starting models for full waveform inversion from wide-aperture data by stereotomography: Geophysical Prospecting, 61, 109-137, doi: 10.1111/j.13652478.2012.01099.x.

Roberts, G., 2000, Wide-angle AVO: 70th Annual International Meeting, SEG, Expanded Abstracts, 134-137.

Rocchi, S., A. Mazzotti, M. Marroni, L. Pandolfi, P. Costantini, D. Di Biase G. Bertozzi, F. Federici, and G. Lo Papa, 2007, Detection of Miocene saucer-shaped sills (offshore Senegal) via integrated interpretation of seismic, magnetic and gravity: Terra Nova, 19, 232-239, doi: 10.1111/j.13653121.2007.00740.x.

Rupert, G. B., and J. H. Chun, 1975, The block move sum normal moveout correction: Geophysics, 40, 17-24, doi: 10.1190/1.1440511.

Shatilo, A., and F. Aminzadeh, 2000, Constant normal-moveout (CNMO) correction: A technique and test results: Geophysical Prospecting, 48, 473-488, doi: 10.1046/j.1365-2478.2000.00190.x.

Stucchi, E., and A. Mazzotti, 2009, 2D seismic exploration of the Ancona landslide (Adriatic Coast, Italy): Geophysics, 74, no. 5, B139-B151, doi: 10.1190/1.3157461.

Taner, M. T., and F. Koehler, 1969, Velocity spectra-digital computer derivation and applications of velocity functions: Geophysics, 34, 859-881, doi: 10.1190/1.1440058.

Trickett, S., 2003, Stretch-free stacking: 73rd Annual International Meeting, SEG, Expanded Abstracts, 2008-2011.

Tygel, M., J. Schleicher, and P. Hubral, 1994, Pulse distortion in depth migration: Geophysics, 59, 1561-1569, doi: 10.1190/1.1443545.

White, R. S., J. R. Smallwood, M. M. Fliedner, B. K. Boslaugh, J. Maresh, and J. Fruehn, 2003, Imaging and regional distribution of basalt flows in the Faeroe-Shetland Basin: Geophysical Prospecting, 51, 215-231, doi: 10.1046/j.1365-2478.2003.00364.x.

Zhang, B., K. Zhang, S. Guo, and K. Marfurt, 2013, Nonstretching NMO correction of prestack time-migrated gathers using a matching-pursuit algorithm: Geophysics, 78, no. 1, U9-U18, doi: 10.1190/geo20110509.1 .

Zhu, X., and G. A. McMechan, 2013, Stretch-free migration imaging condition: Geophysics, 78, no. 4, S203-S210, doi: 10.1190/geo2012-0519.1. 